\title{
Who is More Hands on with Hand-offs? A Comparative Study of Clinical Handovers among Doctors and Nurses in a Tertiary Care Center in India
}

\author{
${ }^{1}$ Parmeshwar Kumar, ${ }^{2} \mathrm{~V}$ Jithesh, ${ }^{3}$ Aarti Vij, ${ }^{4}$ Shakti Kumar Gupta
}

\begin{abstract}
Background: Standardized handovers have been known to improve outcome, reduce error and enhance communication. Few, if any, comparative studies on clinical handovers have been conducted in the India.
\end{abstract}

Objective: To study clinical handover practices among nurses and doctors in a neurosciences center in India.

Design and setting: This descriptive and cross-sectional study was conducted over 4 months in a 200 bedded public sector tertiary care facility in New Delhi, India.

Materials and methods: The handover practices of nurses and resident doctors in a neurology ward were assessed across shifts, weekdays and weekends using a pretested checklist. Ten elements were observed under the categories of time, place, record, process, staff interaction and patient communication. Outcomes were analyzed using z-test, analysis of variance (ANOVA) and Spearman's correlation coefficient.

Results: Three hundred and eighty-two handovers each of nurses and doctors revealed varying adherence for time $(44 \%)$, place $(63 \%)$, documentation $(50 \%)$, process $(78 \%)$, staff interaction (50\%) and patient communication (45\%) related elements with overall compliance being $55 \%$. Doctors fared better only in process elements and bedside handovers; however, only nurses had a statistically significant fall in levels over weekends and in night shifts. Staff interaction and patient communication were positively correlated and bedside handover was negatively related to handover duration in

${ }^{1}$ Assistant Professor, ${ }^{2}$ Lieutenant Colonel, ${ }^{3}$ Professor and Additional Medical Superintendent, ${ }^{4}$ Medical Superintendent

${ }^{1}$ Department of Hospital Administration, All India Institute of Medical Sciences, New Delhi, India

${ }^{2}$ Army Medical Corps, Ministry of Defence, Government of India, New Delhi, India

${ }^{3}$ Department of Hospital Administration; Cardiothoracic and Neurosciences Centre, All India Institute of Medical Sciences New Delhi, India

${ }^{4}$ Department of Hospital Administration, Dr RP Centre for Ophthalmic Sciences, All India Institute of Medical Sciences New Delhi, India

Corresponding Author: Parmeshwar Kumar, Assistant Professor, Department of Hospital Administration, All India Institute of Medical Sciences, New Delhi, India, Phone: 918765977620, e-mail: drpkaiims@gmail.com both groups. No statistically significant difference was found between the two groups when assessed as categories.

Conclusion: Study revealed a need for a system change and standardization of clinical handovers. Greater administrative commitment, use of technology, customized training and leadership development will aid in continuity of care, promote patient safety and ensure better outcomes.

Keywords: Clinical handovers, Shifts, Standardization.

How to cite this article: Kumar P, Jithesh V, Vij A, Gupta SK. Who is More Hands on with Hand-offs? A Comparative Study of Clinical Handovers among Doctors and Nurses in a Tertiary Care Center in India. Int J Res Foundation Hosp Healthc Adm 2015;3(1):33-40.

\section{Source of support: Nil}

Conflict of interest: None

\section{INTRODUCTION}

Clinical handover has been defined as the process of transferring primary authority and responsibility for providing clinical care to a patient from one departing caregiver to one oncoming caregiver. ${ }^{2}$ Nursing handover or shift report is a type of clinical handover that occurs between two shifts of nurses, whereby the specific purpose is to communicate information about patients under the care of nurses. ${ }^{20}$ Multicentric prospective intervention studies have shown that implementation of hand-off programs are associated with reductions in medical errors and improvements in communication, without a negative effect on workflow. ${ }^{32}$ Others have shown differences in handover practices across care- giver type. ${ }^{42}$ Some studies have observed a decrease in length of hospital stay and, therefore, the cost of individual medical visits and fewer referrals. ${ }^{11}$ Though, quality of doctors' handovers is now regarded as a key area for improvement in patient safety; ${ }^{3}$ research on healthcare handovers is mainly on the nursing shift report. ${ }^{18,19}$ However, physician-to-physician handoffs have also been identified as a high-risk area of patient care that can result in serious safety events, near misses, suboptimal patient care, and poor coordination of care. ${ }^{15}$

Few, if any, studies have been conducted in the Indian public hospital scenario regarding clinical handover communication. The authors are not aware of any Indian study that compares nursing and physician handovers. 
Considering the heavier patient load in Indian public sector hospitals, the lack of standardized protocols, it was considered to undertake the study on clinical handover practices in the neurosciences center.

\section{MATERIALS AND METHODS}

This descriptive and cross-sectional study was conducted in a 200 bedded neurosciences center of an apex public sector tertiary care referral hospital in New Delhi, India from January 2014 to April' 2014. Nonparticipant observation was conducted between groups of caregivers as follows:

- Nursing handovers during change of shifts

- Physician handovers between residents during change of shifts.

Morning and night shift handovers were observed in a neurology ward, representative of the neurosciences center, using a pretested checklist. Handovers in the ward were observed during weekdays and weekends as well. In each nursing shift, handover practices in relation to all the patients under the charge of one staff nurse was observed. The nurse handovers were observed in rotation to minimize individual variation. Since, resident handovers occur only in the morning and night shifts, nurse evening shift was excluded to permit comparability. The beds observed for both groups of caregivers were the same to overcome case based variability. Administrative nurses, physician-nurse handovers, consultant hand-overs, interhospital transfer handovers, intensive care unit (ICU) to ward and private ward beds handovers were excluded from the study. The sample size was determined in accordance with the World Health Organization $(\mathrm{WHO})$ guidelines, ${ }^{43}$ using the formula:

$$
\mathrm{N}=(\mathrm{SD} / \delta)^{2} \times \mathrm{p}(1-\mathrm{p})
$$

where $\mathrm{SD}=$ standard deviation at $95 \%$ confidence level, $\delta=$ width of interval, $\mathrm{p}=$ proportion.
The pretested healthcare worker-neutral handover checklist was adopted from the implementation toolkit promulgated by the Australian Medical Association and the United Kingdom National Patient Safety Agency (2004). ${ }^{17,33}$ A pilot test of nursing and resident shift practices was carried out in both the shifts for 1 week. This modified, validated checklist was then used to carry out the study.

The handover checklist consisted of 10 elements under six categories with 10 boxes corresponding to the elements under each category (Table 1). The data collection was undertaken over a period of 40 days to include 5 weekends. The mean value of all the handovers for that shift were taken as representative for that shift. Weekends were also analyzed independently. One way and two way analysis of variance (ANOVA sig: $<0.05$ ), z-test for difference of proportions (sig: $<0.01$ ), and Spearman's correlation coefficient was used to analyze the data using SPSS 22 software.

\section{RESULTS}

The neuroscience center of the tertiary referral center is a seven storied structure with 200 beds including general wards, ICU and private wards. The nursing shift handover in the 30 bedded neurology ward (as in the rest of the hospital as well) selected for the study occurs over three shifts: morning (M), evening (E) and the night shift $(N)$. Since each staff nurse is in-charge of 5 patients, and evening shifts were excluded, in a 24-hour period, 10 handover were observed. Using the formula for sample size calculation: $\mathrm{N}=(\mathrm{SD} / \delta)^{2} \times \mathrm{p}(1-\mathrm{p}), \mathrm{SD}=1.96, \delta$ of $5 \%$ and $\mathrm{p}=50 \%$ (for maximum sample size).

$\mathrm{N}$ was calculated to be 382 . Thus, 382 nursing (191 morning and 191 evening shift handovers) and 382 resident handovers (191 morning and 191 evening shift handovers) were observed.

Table 1: Pretested checklist used as tool for clinical handovers with 10 elements in six categories

\begin{tabular}{|c|c|c|}
\hline Category & Element & Description \\
\hline \multirow[t]{2}{*}{ Time } & 1. Specificity $\square$ & Whether the handover occurs at a specific pre-determined time \\
\hline & 2. Duration $\square$ & $\begin{array}{l}\text { Whether duration of handover has been sufficient to convey relevant } \\
\text { information }\end{array}$ \\
\hline Place & 3. Bedside handover $\square$ & Whether significant part of handover occurring at the bedside \\
\hline Record & 4. Documentation $\square$ & Whether important aspects of the handovers have been recorded \\
\hline \multirow[t]{4}{*}{$\begin{array}{l}\text { Process } \\
\text { (SBAR) }\end{array}$} & 5. Situation $\square$ & $\begin{array}{l}\text { Whether the patient's diagnosis or reason for admission explained to the } \\
\text { oncoming caregiver }\end{array}$ \\
\hline & 6. Background $\square$ & Whether the clinical background or context explained during handover \\
\hline & 7. Assessment $\square$ & $\begin{array}{l}\text { Whether an assessment of current situation is provided and a professional } \\
\text { opinion of the condition given }\end{array}$ \\
\hline & 8. Recommendation $\square$ & $\begin{array}{l}\text { Whether professional recommendation or advice regarding further } \\
\text { management is provided }\end{array}$ \\
\hline Staff interaction & 9. Read-back $\square$ & Whether sufficient interaction including between the caregivers has occurred \\
\hline Patient communication & 10. Counseling $\square$ & Whether relevant information has been conveyed to the patient \\
\hline
\end{tabular}


Outcome was analyzed with regards to the elements in following categories against group of caregivers, shifts, week days and over weekends:

- Time: These included whether handovers occurred at specific pre-determined time and duration. Doctors as well as nurses showed lower compliance over night and weekend for punctuality and the duration was shorter as well (Graph 1). However, the difference over the week end was statistically significant (z-test sig: $0.004)$ only in the case of nurses. The overall adherence was $43 \%$. Two way ANOVA post hoc test revealed that the statistically difference between doctors and nurses was due to lack of handover punctuality (specificity) among doctors (ANOVA sig: 0.023).

- Bedside handover: Over the week and in both shifts, doctors had a greater propensity to conduct handovers at bedside than nurses (compliance nurses: $58 \%$, resident doctors: $67 \%$ ). Handovers that did occurred at bedside had shorter duration. In addition, bedside handovers were more often in both groups at weekend and night shift (Graph 2). One way ANOVA test revealed statistically significant difference only within nurses, bedside handover being significantly higher over the weekends (one way ANOVA sig: 0.03).

- Documentation: Adherence of handover documentation was overall $49 \%$, with nursing documentation marginally higher at $52 \%$ (doctors: $47 \%$ ). Documentation was poorer in both groups during weekends and night shifts; the difference being statistically significant only in the case of doctors $(z=0.004)$.

- Process: In both groups, process elements situation, background, assessment, recommendation (SBAR) were followed the best among all the five categories studied, with doctors performing better (Aggregate; doctors: $83 \%$, nurse: $72 \%$ ). Among nurses, across both

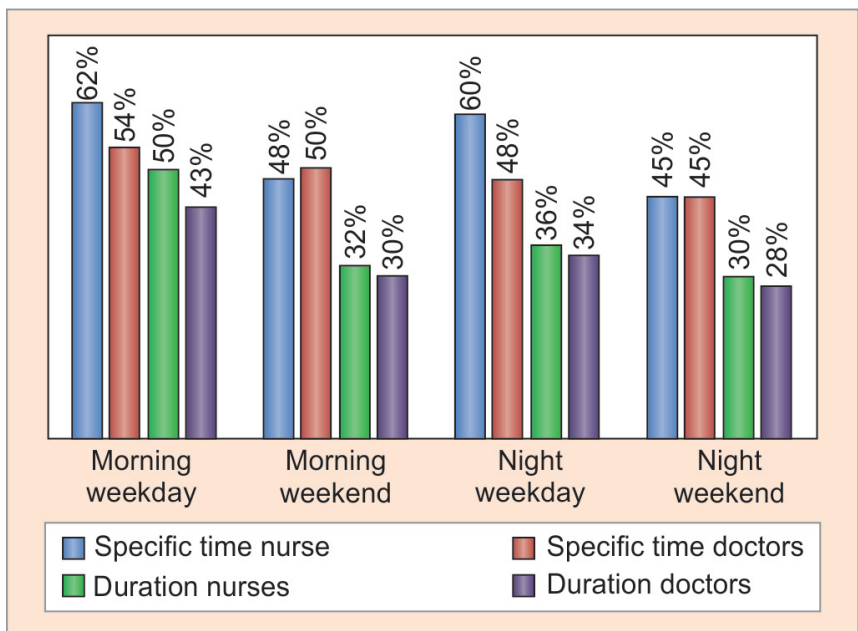

Graph 1: Handover compliance among nurse and resident doctors regarding time elements of specificity (punctuality) and duration is compared over morning and night shift, during weekdays and over weekends shifts, elements of situation (76\%) and background (74\%) had better adherence than assessment $(70 \%)$ and recommendation $(68 \%)$. The reverse was true in case of doctors [situation ( $80 \%)$, background $(82 \%)$, assessment (86\%), recommendation (84.4\%) (Graph 3)]. The difference was statistically significant between doctors and nurse (z-test; sig: 0.004). However, the difference between shifts and over weekends were statistically insignificant for both groups (two way ANOVA sig: 0.454).

- Staff interaction: The overall interaction among all nursing staff during handovers was higher than resident doctors (52.8 and 48\% respectively) (Graph 4). The difference was statistically significant (z-test; sig: 0.006). The task of 'read back' or 'repeat back' by the incoming nurse was being followed less often during the night shifts and weekends, but was not statistically significant. Such a variation was not observed among resident doctors.

- Patient communication: Among all categories, patient communication was given the least priority (44.4\%). However, the nursing staff fared significantly better than resident doctors [(nurse: $48 \%$, doctors: $42 \%$ ) (Graph 4) (z-test; sig: 0.004)]. Although, communication was observed to be lower in the weekends, it may have been a chance finding.

Strong negative correlation was observed between weekend shift and elements related to time (Spearman's coefficient of correlation-0.764) (Table 2) only in the case of nursing staff. Strong negative relationship was also observed between bedside handovers and duration of handover (Spearman's correlation coefficient -0.689 for nurses and -0.568 for doctors) for both groups (Graph 5). Positive correlation was observed with staff interaction and patient communication (Spearman coefficient: +0.569

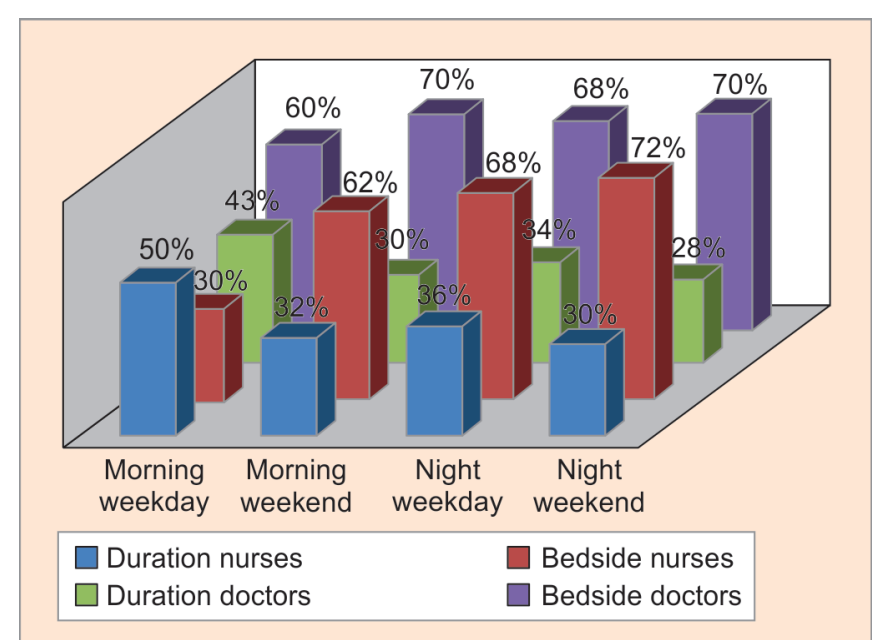

Graph 2: The relationship between the duration of handover and bedside handover compliance among nurses and doctors showing a dip over night shifts and weekends only among nurses 


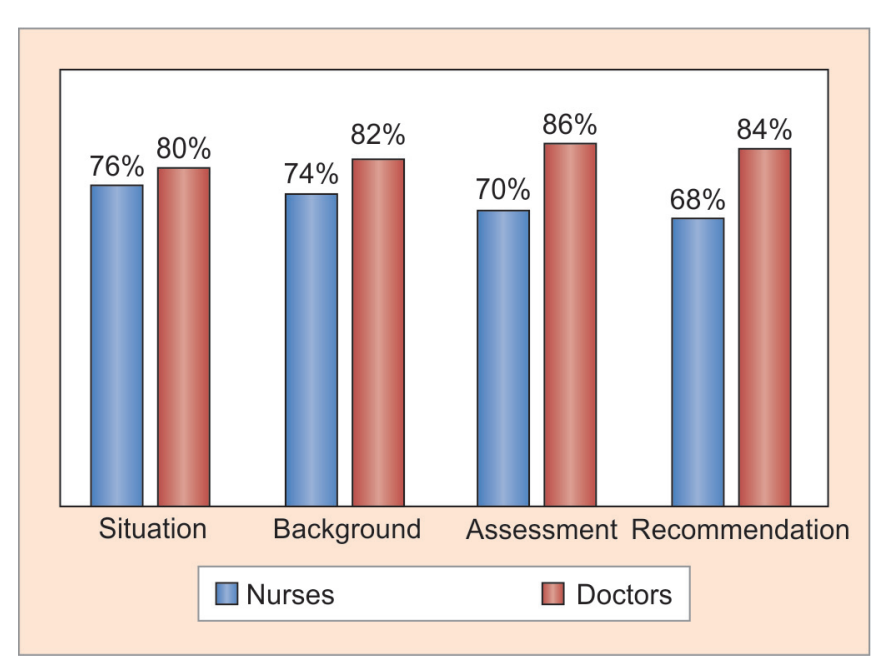

Graph 3: Comparison of process elements; situation, background, assessment and recommendation, between nurses and doctor showed doctors adhered better with SBAR

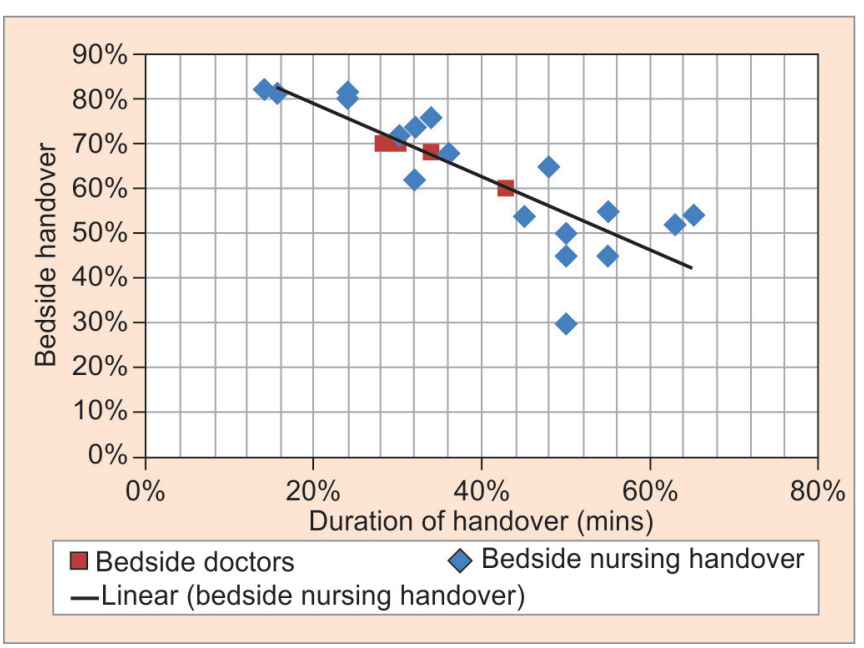

Graph 5: Strong negative correlation between conducting handovers at bedside and duration of handover observed for both doctors and nurses

+0.562 ) for both groups, nurses as well as doctors (Graph 6). However, no correlation was observed between shifts and weekends among doctors. Compliance with respect to all the categories between groups offered similar trends over shifts and days (Graph 7). The difference was not statistically different between the groups ( $\mathrm{z}$-value $=0.023$ ). The overall compliance was 55\%.

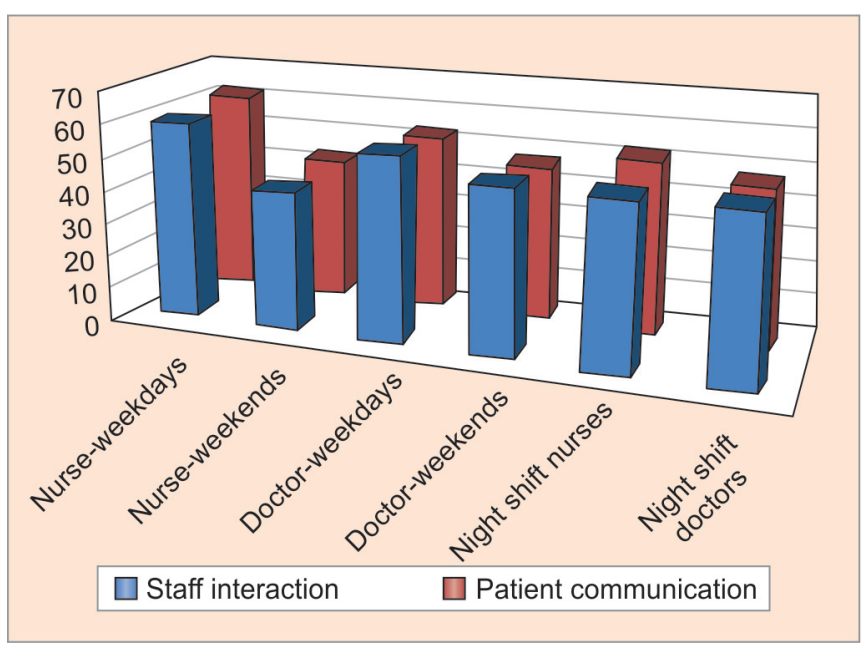

Graph 4: Staff interaction between nurses and their communication with patients was found better than that by doctors

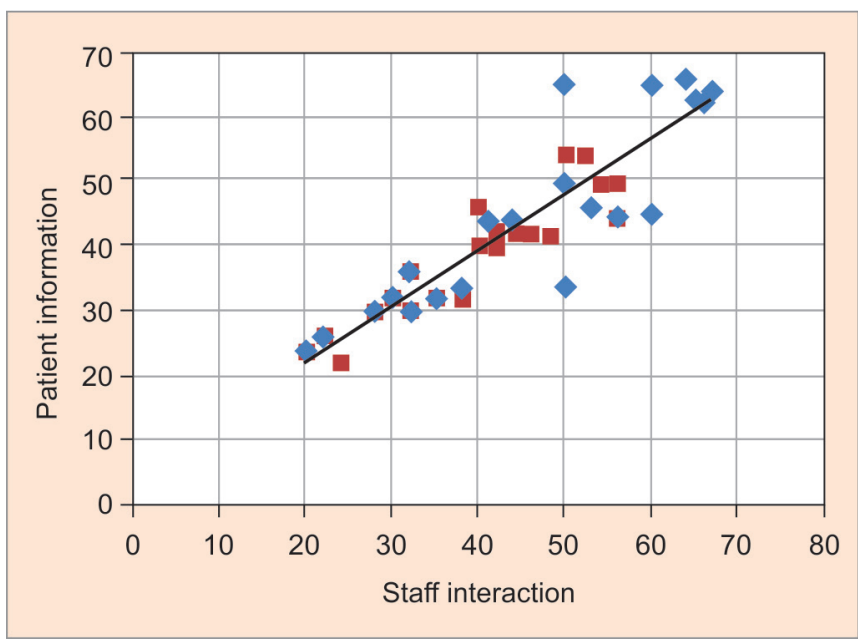

Graph 6: Positive correlation during handovers between interaction among nursing staff (blue dots) and among resident doctors (red dots) and patient communication

\section{DISCUSSION}

Shift handovers are essential, not only for maintaining the continuity and quality of care but also in planning. ${ }^{22,27}$

The objective of conducting a study of handover practices among nursing and resident doctors at the neurosciences center was not a competitive comparison, rather a measure of gaps in the groups. It was hoped

Table 2: Correlation between various variables of the caregiver groups

\begin{tabular}{llll}
\hline & & Statistical analysis: Spearman's rank correlation coefficient \\
\cline { 2 - 4 } Sl. no. & Caregiver group & Variables & Spearman's coefficient of correlation \\
\hline 1. & Nurses & Weekend shifts ${ }^{*}$ time & -0.764 \\
2. & Nurses & Duration* bedside handover & -0.689 \\
3. & Resident doctor & Duration* bedside handover & -0.568 \\
4. & Nurses & Weekend shifts * process & -0.221 \\
5. & Nurses & Night shifts *staff read back & 0.311 \\
6. & Nurses & Read back* patient information & 0.569 \\
7. & Resident doctor & Read back* patient information & 0.562 \\
\hline
\end{tabular}

*Variables have been analyzed using Spearman's coefficient of correlation. Positive and negative values represent positive and negative correlations respectively. Only significant correlations have been tabulated 


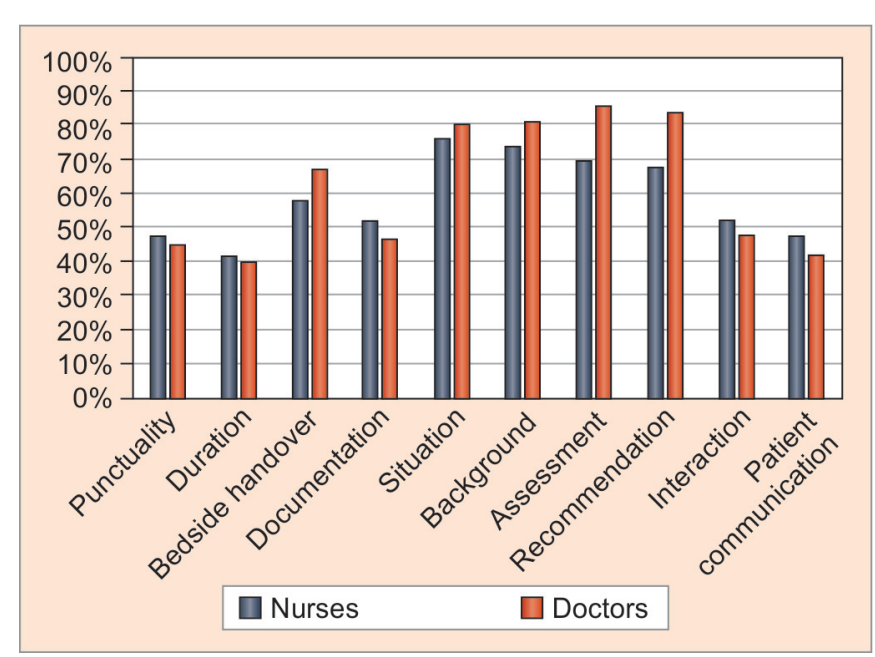

Graph 7: Overall compliance among nurses and doctors for 10 handover elements across all shifts and weeks did not reveal statistically significant difference at a macro level

that a validated healthcare neutral tool provided an opportunity to assess the deficiencies in a system that functions with an unstructured handovers.

Studies indicate the importance of duration and specific time for handovers. ${ }^{29}$ Yet, significant deficiencies were found, especially during the night shifts and weekends among nurses. The findings collaborate those of other studies. ${ }^{37}$ Low morning compliance probably reflects delay in arrival of the morning shift oncoming practitioner, busy morning duty corresponding with doctors rounds and nurse fatigue factor due to night duty. The weekend fall of compliance may be a result of lack of supervision (absence of administrative nurses) and generalized fall in diligence observed in other studies as well. The lack of punctuality among doctors in our study seems to validate other study findings which suggest that in healthcare, most handovers do not occur under ideal conditions, and clinicians may fail to allocate enough time to appropriately transfer patient data. ${ }^{38}$ It is likely that the 12 hours shift of doctors and the charge of the entire 30 beds as against an 8 hours shift with six beds charge among nurses would have affected both, the specificity and duration of handovers. That there was no variation between shifts, or weekends among doctors, point to a more systemic deficiency. However, shorter duration does not necessarily reflect poorer quality of handover or the lack of time. This study revealed that bedside handovers were inversely related to the duration of handover (Graph5) for both groups and since more doctors preferred to conduct handovers at bedside, this may have led to their shorter handover. This also validates the findings of other studies which suggest that bedside handover takes less time. ${ }^{23}$ This may be a subconscious adaptation by both groups to save time; doctors since they have more patients and longer shifts and nurses during night shifts and weekends. Bedside handover has been known to facilitate a partnership model in medication communication bring nursing team, together, providing a patient centered dimension of handovers, with an additional advantage of patients providing key essential information and an opportunity to participate actively in the process of their treatment. ${ }^{6,10,21}$ In our study, poor levels of handover documentation in both groups (52\%) was seen, despite conclusive evidence from other researchers suggesting that only $2.5 \%$ of patient information is retained using verbal only handover method, $85.5 \%$ is retained using verbal with note taking while $99 \%$ was retained using a printed handover. ${ }^{5}$

Documentation is this only element that showed a significant variation over weekends and night shifts among doctors calling for administrative intervention. It has also been suggested that the lack of documentation among doctors have contributed to the problems leading to this adverse event. ${ }^{39}$

The findings of a qualitative case study in two hospitals indicate that SBAR may aid in schema development that allows rapid decision making, provide social capital and legitimacy for less-tenured nurses, and reinforce a move toward standardization in the nursing profession. ${ }^{41,42}$ A prospective interventional study among doctors reveals that SBAR improves communication and safety climate and decreases incident reports due to communication errors. ${ }^{30}$ Encouragingly, our study revealed that process based elements had an overall better compliance in both groups, and SBAR, the core aspect of handover communication, was better adhered to by doctors, thus perhaps being a surrogate indicator for good clinical care. However, the fact that nurses lay less stress upon explaining patient care back ground and their own assessment of the patient, renders handover mechanical and not evdience based. This ritualized handover has been studied to be ineffective and a discourse of anxiety among nurses..$^{12}$ Authors have given various modification to support flexible adaptation, such as the standardized protocols identify-situation-observations-backgroundagreed plan-read (ISOBAR) back, or more recently the- 'hand me an isobar. ${ }^{28,44}$ No localized modification was observed in any of the handovers in our study. A weak negative correlation between week end shift and SBAR continues to point toward the possibility of the weekend effect. Detailed analysis of the content was beyond the scope of the study.

The importance of personnel interaction, especially read back is emphasised by WHO. However, this study revealed a poor level of compliance with read backs (52.8\%) especially among doctors and was validated by other studies. ${ }^{1,14}$ We also confirmed findings from other studies that read-backs are relatively infrequently 
used during patient handovers in all caregiver groups. ${ }^{31}$ Studies also suggest that nurses are less likely than physicians to be assertive, interject and disagree with the previous caregiver, especially when an error was believed to have occurred., ${ }^{9,34}$ This finds reflection in our own study where the assement and recommendation (situations where interjection is likely to occur) were paradoxically lower among nurses despite readback being higher in the group. Positive correlation of nursing read backs with night shifts may indicate an absence other distractors, such as consultant rounds, morning investigations, etc. Surprisingly, our study revealed that greater percentage of bedside handover does not necessarily translate into better staff communication (as in the case of doctors). Further, though contents were not analyzed, observers noted that handovers were formulaic, partial and, used abbreviations and jargon. This has been observed in others studies which suggest that doctor interaction was cryptic, given at high speed often required socialized knowledge to interpret. ${ }^{26}$ Thus, it brings out the importance of leadership and training in implementation of better handover practices, as enumerated by several studies. ${ }^{24}$

The patient and family are the only constant and are thus in a position to play a critical role in ensuring continuity of care. ${ }^{8,42}$ Engaging patients are sometimes made more difficult due to low health literacy. ${ }^{40}$ This is especially true in the Indian context where healthcare workers have a paternalistic view of patients and inequalities in levels of empowerment and opportunity affect medical decision making. ${ }^{36}$ Teach-back is a technique used by caregivers to ensure that the patient has understood the information provided. ${ }^{35}$ Our study showed that despite the type of patient clienetele in public sector Indian hospitals requiring better counseling, handover patient communication had the least adherence among all elements. Doctors engaged with patients less frequently than nurses. Though, the fact that the residents had nearly 5 times the number of beds to manage than nurses and had also academic and independent work commitment (e.g. OPD duties) renders such a comparison unequal; other complex factors besides time, such as language barriers, sociocultural norms may also be at play. ${ }^{7,25}$ Encouragingly though, greater staff interaction was found to be directly related to better patient communication (Graph 6). Thus, an increased impetus on physician to physician interaction during handover is likely to improve communication with patients as well. A study of neurosciences residents concludes that thereneeds to bemore focused education devoted to learning effective patient-care hand-offs in neurosurgical training programs, thus further emphasizing the need for training. ${ }^{4}$
This study had several limitation including that of Hawthorne effect on the staff performing handovers in the presence of the author. ${ }^{16}$ The fact that resident doctors and nurses had different shift hours and beds to manages reduces the comparability. Further, the role of content of handover and patients and family members was not included in the study. Moreover, the large influence of extraneous factors, such as type of clinical environment, experience, culture of leadership, specialty, case mix, technology and local policies, cannot be underestimated. At a macro level, the study did not reveal a statistically significant difference between the groups of healthworkers when all 10 elements were assessed together. Perhaps, more tellingly, the findings being consistent across weekdays and shifts, brought out consistent gaps clearly pointing out system-based deficiencies and need for profession specific training.

\section{CONCLUSION}

This study was undertaken to assess and possibly compare the clinical handover practices among nurses and resident doctors in an apex neurosciences center in India. Relatively, inferior handover practices across all categories, in both groups, calls for a systems approach and greater administrative commitment for standardized handovers. Deficient physician interaction and patient communication by doctors needs to be addressed. Better performance among process elements across the spectrum is encouraging, however, nurses continued to give less importance on handing over their own assessment and recommendation to the incumbent, emphasizing the need for training. Since, better interaction showed direct relation with patient communication, promoting the former is likely to result in overall improvement.

The fact that no significant difference was found between the two groups at a macro level is itself a marker for eschewing the traditional one-size-fits-all simplistic solutions. Therefore, viewing it from the administrative lens, training in both groups has to be customized to plug the gaps identified. While this contextual study revealed an urgent need for standardizing handovers protocol, the use of technology, such as electronic handovers, role of leadership and training cannot be overemphasized. ${ }^{13}$ Future studies need to focus on the postinterventional analysis, content of handovers, perception of residents and staff, and the role of leadership in handovers. This will assist in continuity of care, promote patient safety and ensure better outcomes.

\section{REFERENCES}

1. Apker J, Mallak LA, Applegate EB, et al. Exploring emergency physician-hospitalist handoff interactions: development of 
the handoff communication assessment. Ann Emerg Med 2010 Feb;55(2):161-170.

2. Australian Commission on Safety and Quality in Health Care-Clinical Handover. ACSQHC, 2012. Available at: www.safetyandquality.gov.au/our-work/clinical-communications/clinical-handover Accessed on: 18 Dec 2013.

3. Australian Medical Association Limited. Safe handover: safe patients. Guidance on clinical handover for clinicians and managers. 2006. Available at: http://www.ama.com.au/ node/4064 (Accessed on: 30 Sep 2013).

4. Babu MA, Nahed BV, Heary RF. Investigating the scope of resident patient care handoffs within neurosurgery. PLoS One 2012;7(7):e41810.

5. Bhabra G, Mackeith S, Monteiro P, Pothier DD. An experimental comparison of handover methods. Ann R Coll Surg Engl 2007 Apr;89(3):298-300.

6. Bradley S, Mott S. Handover: faster and safer. J Clin Nurs 2014 Jul;23(13-14):1927-1936.

7. Burbano O'Leary SC, Federico S, Hampers LC. The truth about language barriers: one residency program's experience. Pediatrics 2003;111(5 Pt 1):e569-e573.

8. Cahill J. Patient's perceptions of bedside handovers. J Clin Nurs 1998 July;7(4):351-359.

9. Cavanaugh SJ. The conflict management style of staff nurses and nurse managers. J Adv Nurs 1991 Oct;16(10):1254-1260.

10. Chaboyer W, McMurray A, Johnson J, Hardy L, Wallis M, Sylvia Chu FY. Bedside handover: quality improvement strategy to 'transform care at the bedside'. J Nurs Care Qual 2009 Apr-Jun;24(2):136-142.

11. Chio A, Montuschi A, Cammarosano S. ALS patients and caregivers communication preferences and information seeking behaviour. Eur J Neurol 2008 Jan;15(1):55-60.

12. Evans AM, Pereira DA, Parker JM. Discourses of anxiety in nursing practice: a psychoanalytic case study of the changeof-shift handover ritual. Nurs Inq 2008 Mar;15(1):40-48.

13. Govier M, Medcalf P. Living for the weekend: electronic documentation improves patient handover. Clin Med 2012 Apr;12(2):124-127.

14. Greenstein EA, Arora VM, Staisiunas PG, et al. Characterising physician listening behaviour during hospitalist handoffs using the HEAR checklist. BMJ Qual Saf 2013 May;22(3): 203-209.

15. Hinami K, Farnan JM, Meltzer DO, Arora VM. Understanding communication during hospitalist service changes: a mixed methods study. J Hosp Med 2009 Nov;4(9):535-540.

16. Holden JD. Hawthorne effects and research into professional practice. J Eval Clin Pract 2001 Feb;7(1):65-70.

17. Implementation toolkit: standard key principles for clinical handover. Available at: http://www.archi.net.au/documents/ resources/qs/clinical/clinical-handover/implementationtoolkit.pdf. Accessed on: 26 Nov 2013.

18. Kerr MP. A qualitative study of shift handover practice and function from a sociotechnical perspective. J Adv Nurs 2002 Jan;37(2):125-134.

19. Lally S. An investigation into the functions of nurses' communication at the inter-shift handover. J Nurs Manag 1999 Jan;7(1):29-36.

20. Lamond D. The information content of the nurse change of shift report: a comparative study. J Adv Nurs 2000 Apr;31(4):794-804.
21. Liu W, Manias E, Gerdtz M. Medication communication between nurses and patients during nursing handovers on medical wards: a critical ethnographic study. Int J Nurs Stud 2012 Aug;49(8):941-952.

22. Liukkonen A. The content of nurses' oral shift reports in homes for elderly people. J Adv Nurs 1993 Jul;18(7):1095-1100.

23. McMurray A, Chaboyer W, Wallis M, Fetherston C. Implementing bedside handover: strategies for change management. J Clin Nurs 2010 Sep;19(17-18):2580-2589.

24. Meissner A, Hasselhorn HM, Estryn-Behar M, Nézet O, Pokorski J, Gould D. Nurses' perception of shift handovers in Europe: results from the European Nurses' early exit study. J Adv Nurs 2007 Mar;57(5):535-542.

25. Ngo-Metzger Q, Massagli MP, Clarridge BR, et al. Linguistic and cultural barriers to care. J Gen Intern Med 2003 Jan;18(1):44-52.

26. Payne S, Hardey M, Coleman P. Interactions between nurses during handovers in elderly care. J Adv Nurs 2000 Aug;32(2):277-285.

27. Philpin S. 'Handing over': transmission of information between nurses in an intensive therapy unit. Nurs Crit Care 2006 Mar-Apr;11(2):86-93.

28. Porteous JM. iSoBAR — concept and handover checklist: the national clinical handover initiative. Med J Aust 2009 Jun 1;190(11 suppl):S152-156.

29. Randell R, Wilson S, Woodward P. The importance of the verbal shift handover report: a multi-site case study. Int J Med Inform 2011 Nov;80(11):803-812.

30. Randmaa M, Mårtensson G, Leo Swenne C, Engström M. SBAR improves communication and safety climate and decreases incident reports due to communication errors in an anaesthetic clinic: a prospective intervention study. BMJ Open 2014 Jan;4(1):e004268.

31. Rayo MF, Mount-Campbell AF, O'Brien JM, et al. Interactive questioning in critical care during handovers: a transcript analysis of communication behaviours by physicians, nurses and nurse practitioners. BMJ Qual Saf 2014 Jun;23(6): 483-489.

32. Root causes of sentinel events, all categories Oakbrook, IL: Joint Commission, 2006. Available at: http://www.jointcommission.org/NR/rdonlyres/FA465646-5F5F-4543-AC8FE8AF6571E372/0/root_cause_se.jpg, Accessed on: 12 Sep 2013.

33. Safe Handover: Safe Patients' Guideline by the United Kingdom National Patient Safety Agency (2004). Available at: http://bma.org.uk/media/files/pdfs/practical\%2advice $\% 20$ at $\% 20$ work/contracts/safe $\% 20$ handover $\% 20$ safe $\%$ 20patients.pdf. Accessed on 12 Dec 2013.

34. Sayre MM, McNeese-Smith D, Leach LS, et al. An educational intervention to increase 'Speaking-Up' behaviors in nurses and improve patient safety. J Nurs Care Qual 2012 Apr-Jun;27(2):154-160.

35. Schillinger $\mathrm{D}$, et al. Closing the loop: physician communications with diabetic patients who have low health literacy. Arch Intern Med 2003 Jan 13;163(1):83-90.

36. Seetharam S, Zanotti R. Patients' perceptions on healthcare decision making in rural India: a qualitative study and ethical analysis. J Clin Ethics 2009 Summer;20(2):150-157.

37. Sexton A, Chan C, Elliott $M$, et al. Nursing handovers: do we really need them? J Nurs Manag 2004 Jan;12(1):37-42. 
38. Sharit J, McCane L, Thevenin DM, Barach P. Examining links between sign-out reporting during shift changeovers and patient management risks. Risk Anal 2008 Aug;28(4): 969-981.

39. Shojania KG, Fletcher KE, Saint S. Graduate medical education and patient safety: a busy- and occasionally hazardousintersection. Ann Intern Med 2006 Oct 17;145(8):592-598.

40. The ABCs of health literacy, Chicago. American Medical Association. Available at: http://www.ama-assn.org/ama1/ pub/upload/mm/15/health_literacy.doc. Accessed on 11th Jan 2014.

41. Vardaman JM, Cornell P, Gondo MB, Amis JM, TownsendGervis M, Thetford C. Beyond communication: the role of standardized protocols in a changing healthcare environment. Healthcare Manage Rev 2012 Jan-Mar;37(1): 88-97.

42. Whale $Z$. The participation of hospital nurses in the multidisciplinary ward round on a cancer-therapy ward. J Clin Nurs 2004 Feb;2(3):155-163.

43. World Health Organization. Health Research Methodology. 2nd ed. Manila: WHO Regional Office for the Western Pacific, 2001.

44. Yee KC, Wong MC, Turner P. 'Hand me an isobar': a pilot study of an evidence-based approach to improving shift-toshift clinical handover. Med J Aust 2009 Jun 1;190 (11 Suppl): S121-124. 